\title{
Erlotinib-Related Spontaneous Pneumothorax in Patient with Primary Lung Cancer
}

\author{
Hae-Seong Nam, M.D., Hyeon-Jeong Lee, M.D., Min Su Kim, M.D., Sung Wook Park, M.D., Sang-Hoon Jeon, \\ M.D., Jae Hwa Cho, M.D., Seung Min Kwak, M.D., Hong Lyeol Lee, M.D., Jeong-Seon Ryu, M.D. \\ Division of Pulmonary and Critical Care Medicine, Department of Internal Medicine, Inha University Hospital, Inha University \\ School of Medicine, Incheon, Korea
}

Spontaneous pneumothorax (SPTx) associated with primary lung cancer is quite rare, but has been reported as the initial presentation or a complication of disease progression. Moreover, chemotherapy-related SPTx in primary lung cancer occurs at a very low frequency, accounting for less than $0.05 \%$ of all cases. Here, we report the first case of erlotinib-related SPTx in a patient with advanced lung adenocarcinoma in Korea. After 3 cycles of cisplatin-based chemotherapy as first-line therapy, erlotinib was administered as second-line treatment. Asymptomatic SPTx accompanied by a significant decrease in tumor size was observed in the left lung 7 weeks later. The patient received continuous administration of erlotinib, without additional treatment. This case showed that SPTx can occur in patients with primary lung cancer receiving erlotinib, and asymptomatic chemotherapy-related SPTx in primary lung cancer may not require therapeutic intervention.

Key Words: Pneumothorax; Lung neoplasm; erlotinib

\section{Introduction}

Spontaneous pneumothorax (SPTx) is a relatively rare clinical presentation in patients with primary lung cancer. It has been reported mainly as the initial presentation of lung cancer or a complication of disease progression $^{1,2}$. Especially, chemotherapy-related SPTx in primary lung cancer have been reported by sporadic cases $^{2-4}$. Here, we describe the first case of a patient with adenocarcinoma of the lung who presented with SPTx after erlotinib administration, the first reported in Korea.

Address for correspondence: Jeong-Seon Ryu, M.D. Division of Pulmonary, Department of Internal Medicine, Center for Lung Cancer, Inha University Hospital, Inha University School of Medicine, 7-206, Shinheung-dong 3-ga, Jung-gu, Incheon 400-711, Korea

Phone: 82-32-890-3738, Fax: 82-32-882-6578

E-mail: jsryu@inha.ac.kr

Received: Aug. 12, 2010

Accepted: Oct. 1, 2010

\section{Case Report}

An otherwise healthy 48-year-old Korean woman was admitted to our hospital with a 4-month history of dry cough and dyspnea. She had never smoked.

The results of physical examination and laboratory tests were normal. No endobronchial lesions were noted on bronchoscopic examination. Polymerase chain reaction and microscopic examinations (acid-fast bacillus smear and culture) with bronchial washing or sputum were negative for Mycobacterium tuberculosis.

A chest computed tomography (CT) scan showed diffuse consolidation in the left upper lobe of the lung, consolidation and ground glass opacities (GGOs) in the left lower lobe, and tiny consolidations and GGOs in the right lower lobe. Pathology results from a specimen obtained by CT-guided fine-needle biopsy of the consolidation in the left lower lobe showed a well-differentiated adenocarcinoma. The other primary lesion was not found by positron emission tomography-CT (PETCT) scan. Brain magnetic resonance imaging revealed multiple brain metastases. She was administered three 
HS Nam et al: Erlotinib-related spontaneous pneumothorax

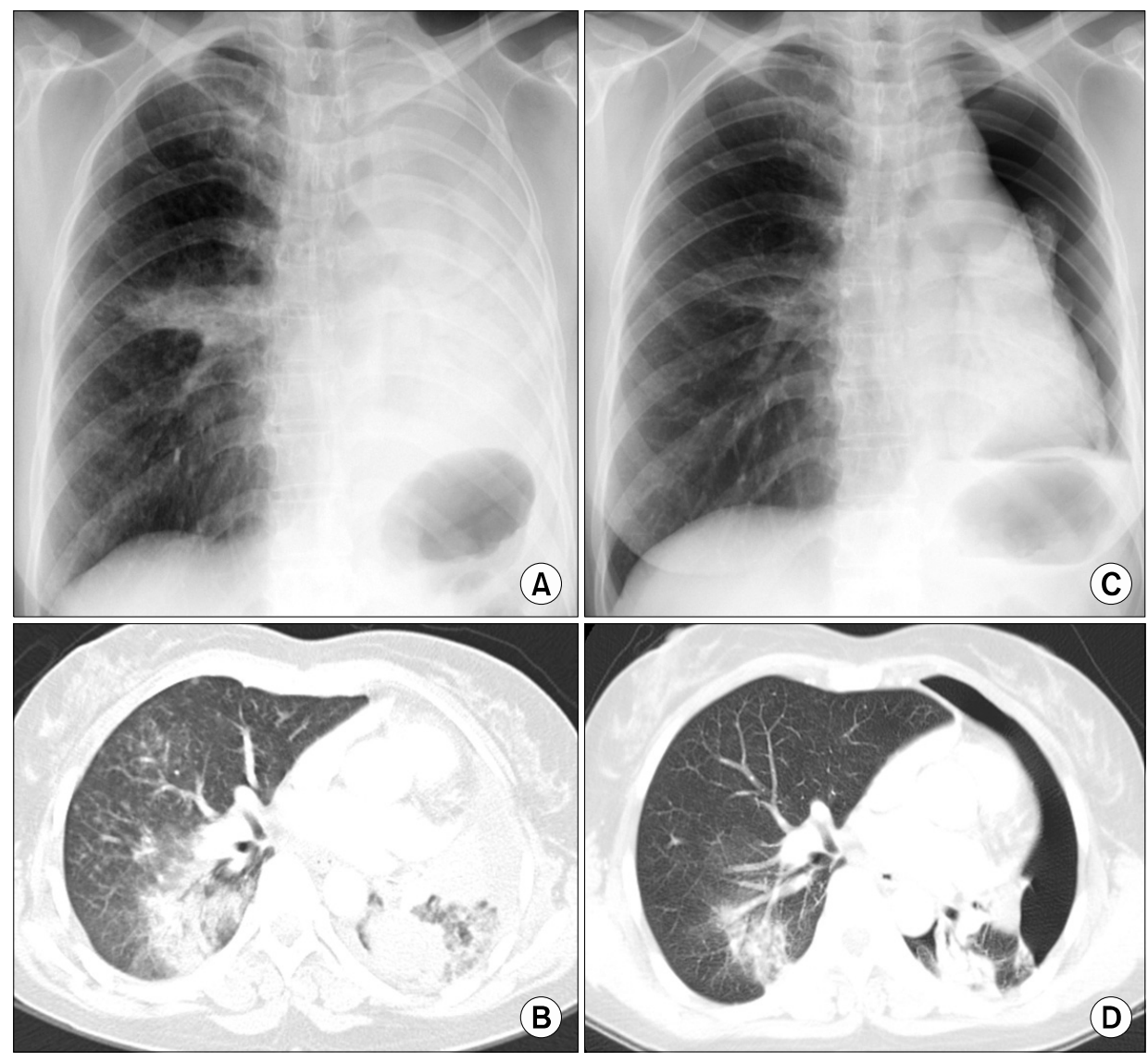

Figure 1. Chest X-ray and computed tomography were performed before treatment with erlotinib (A, B). After 7 weeks of erlotinib administration, a spontaneous pneumothorax was first confirmed in the left lung; the consolidations and ground glass opacities in the lung showed marked reductions in size with erlotinib treatment (C, D).

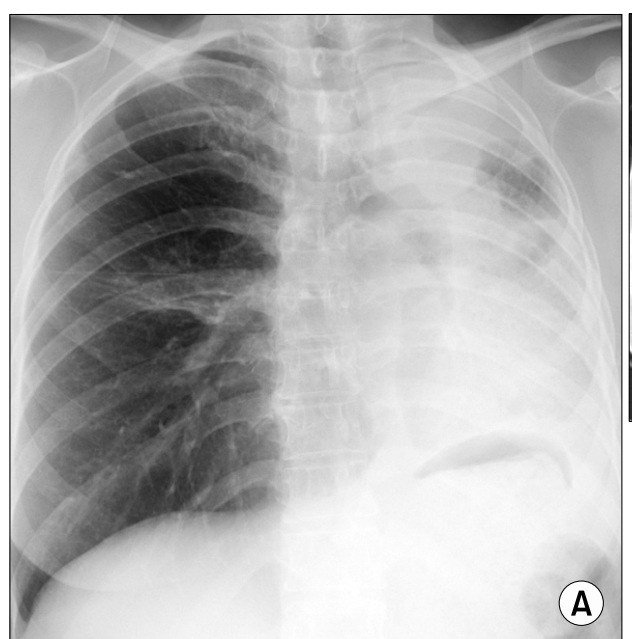

(A)

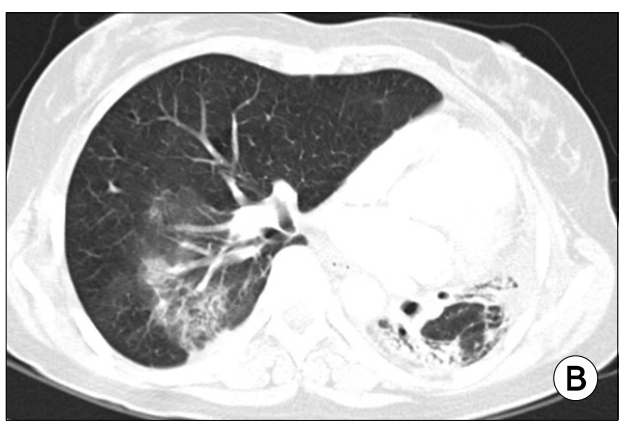

Figure 2. Nine weeks after spontaneous pneumothorax, the left pneumothorax showed spontaneous improvement in chest X-ray (A) and computed tomography (B). 
cycles of cisplatin-based doublets chemotherapy, which showed no effect. After three cycles of cisplatin-based doublets chemotherapy, a chest X-ray (Figure 1A) revealed total opacification of the left lung and right middle lobe infiltration. A chest CT scan (Figure 1B) showed this to be secondary to diffuse consolidations and GGOs in the left lung, and small multiple nodules and consolidations in the right lower lobe. As she had never smoked and tumor biopsy specimens showed a deletion in exon 19 of the epidermal growth factor receptor (EGFR), second-line chemotherapy with erlotinib (daily $150 \mathrm{mg}$ ) was begun.

After 7 weeks, she was asymptomatic at a routine checkup at our outpatient clinic, but her chest X-ray (Figure 1C) demonstrated left SPTx. She reported no history of trauma. Chest CT (Figure 1D) indicated that the consolidations or GGOs in the lung had been markedly reduced in size by erlotinib without severe side effects, except for the SPTx. Thus, the SPTX was observed without any therapeutic intervention. Nine weeks later, chest X-ray (Figure 2A) and CT (Figure 2B) taken at the outpatient clinic revealed spontaneous improvement of the left pneumothorax, even though erlotinib administration had been continued.

\section{Discussion}

SPTx associated with malignant pulmonary neoplasm is rare. It has been reported as a first sign of lung involvement for certain tumors or as a complication arising from radiotherapy or chemotherapy in patients suffering from a variety of malignancies ${ }^{5-8}$. In general, chemotherapy-related SPTx has been reported in chemosensitive tumors, particularly sarcomas or germ cell tumors, with multiple lung metastases ${ }^{6-8}$. However, SPTx associated with primary lung cancer occurs at a very low frequency. Lai et al. ${ }^{2}$ reported that $18(0.32 \%)$ of 5,567 patients with primary lung cancer had SPTX as a complication, and only two (0.04\%) of these cases occurred after chemotherapy. Rarely, sporadic cases of SPTx have been reported associated with chemotherapy in primary lung cancer ${ }^{2-4}$. To the best of our knowledge, this is the first case of erlotinib-related SPTx in a patient with primary lung cancer in Korea.

The mechanism of chemotherapy-related SPTx is largely elusive; however, various mechanisms have been proposed ${ }^{4,6,8}$. For example, rapid tumor lysis or tumor tissue necrosis due to chemotherapy may directly induce the formation of fistulas. Alternatively, there may be underlying subpleural bullae or blebs that rupture after chemotherapy.

Erlotinib is a small-molecule tyrosine kinase inhibitor directed against the EGFR. The presence of an EGFR mutation or of clinical factors such as adenocarcinoma or never having smoked is associated with the response to erlotinib?. Taken together with the temporal relationship between erlotinib administration and radiological regression of the tumors, this was likely the etiology in the patient described here, as she had no history of smoking, was positive for EGFR mutation, had no previous history of lung disease, and lacked any other relevant history. Apoptosis of subpleural tumor cells, induced by tyrosine kinase inhibitors such as erlotinib or gefitinib that are directed against $\mathrm{EGFR}^{10}$, probably induces the formation of bronchopleural fistulas. Therefore, we postulated that erlotinib induced rapid tumor lysis and necrosis of peripherally located tumor tissues, leading to SPTx.

Generally, treatment of chemotherapy-related SPTx involves closed chest tube insertion ${ }^{8}$. In addition, chemical pleurodesis or surgical intervention may be considered in patients with recurrent and/or bilateral chemotherapy-related $\mathrm{SPTx}{ }^{6,7}$. However, some chemotherapyrelated SPTx in patients with primary lung cancer, particularly those responding to chemotherapy for lung cancer, improve spontaneously, without any therapeutic intervention, while continuing chemotherapy ${ }^{3,4}$. The present case showed also that asymptomatic SPTx accompanied by significant decrease in the size of the tumor resolved spontaneously, without any therapeutic intervention, during continued erlotinib administration. These cases suggest that the tissue repair process can continue despite chemotherapy for lung cancer. Further studies are needed to understand the cause of sponta- 
neously resolving chemotherapy-related SPTx.

In summary, this case showed that SPTx can occur in patients with primary lung cancer receiving erlotinib. Asymptomatic chemotherapy-related SPTx in primary lung cancer serves as a reminder to clinicians that it may spontaneously improve with no therapeutic intervention, and that it may represent an indirect measure of response to chemotherapy.

\section{Acknowledgements}

This study was supported by INHA UNIVERSITY Research Grant (INHA-40900-01).

\section{References}

1. Steinhäuslin CA, Cuttat JF. Spontaneous pneumothorax: a complication of lung cancer? Chest 1985;88:709-13.

2. Lai RS, Perng RP, Chang SC. Primary lung cancer complicated with pneumothorax. Jpn J Clin Oncol 1992; 22:194-7.

3. O'Connor BM, Ziegler P, Spaulding MB. Spontaneous pneumothorax in small cell lung cancer. Chest 1992; 102:628-9.

4. Mori M, Nakagawa M, Fujikawa T, Iwasaki T, Kawamura T, Namba Y, et al. Simultaneous bilateral sponta- neous pneumothorax observed during the administration of gefitinib for lung adenocarcinoma with multiple lung metastases. Intern Med 2005;44:862-4.

5. Dines DE, Cortese DA, Brennan MD, Hahn RG, Payne WS. Malignant pulmonary neoplasms predisposing to spontaneous pneumothorax. Mayo Clin Proc 1973;48: 541-4.

6. Biran H, Dgani R, Wasserman JP, Weissberg D, Shani A. Pneumothorax following induction chemotherapy in patients with lung metastases: a case report and literature review. Ann Oncol 1992;3:297-300.

7. Fenlon HM, Carney D, Breatnach E. Case report: bilateral recurrent tension pneumothorax complicating combination chemotherapy for soft tissue sarcoma. Clin Radiol 1996;51:302-4.

8. Stein ME, Shklar Z, Drumea K, Goralnik L, Ben-Arieh Y, Haim N. Chemotherapy-induced spontaneous pneumothorax in a patient with bulky mediastinal lymphoma: a rare oncologic emergency. Oncology 1997;54: 15-8.

9. Tsao MS, Sakurada A, Cutz JC, Zhu CQ, Kamel-Reid $\mathrm{S}$, Squire J, et al. Erlotinib in lung cancer - molecular and clinical predictors of outcome. $\mathrm{N}$ Engl $\mathrm{J}$ Med 2005;353:133-44.

10. Moyer JD, Barbacci EG, Iwata KK, Arnold L, Boman $\mathrm{B}$, Cunningham $\mathrm{A}$, et al. Induction of apoptosis and cell cycle arrest by CP-358,774, an inhibitor of epidermal growth factor receptor tyrosine kinase. Cancer Res 1997;57:4838-48. 